\title{
Asking If the Existence of Vacuum Energy to Keep Computational “Bits” Present at Start of Cosmological Evolution, Even If Spatial Radius Goes to Zero, Not Planck Length, Is Possible
}

\author{
Andrew Beckwith \\ Department of Physics, Chongqing University, Chongqing, China \\ Email: abeckwith@uh.edu
}

Received 10 December 2015; accepted 15 April 2016; published 18 April 2016

Copyright (C) 2016 by authors and Scientific Research Publishing Inc.

This work is licensed under the Creative Commons Attribution International License (CC BY). http://creativecommons.org/licenses/by/4.0/

\section{(c) (i) Open Access}

\begin{abstract}
When initial radius $R_{\text {initial }} \rightarrow 0$ if Stoica actually presents Einstein equations in a formalism which remove the big bang singularity pathology, then the reason for Planck length no longer holds. We present entanglement entropy in the early universe with a shrinking scale factor, due to Muller and Lousto, and show that there are consequences due to initial entangled $S_{\text {Entropy }}=0.3 r_{H}^{2} / a^{2}$ for a time dependent horizon radius $r_{H}$ in cosmology, with (flat space conditions) $r_{H}=\eta$ for conformal time. Even if the 3 dimensional spatial length goes to zero, this construction preserves a minimum non-zero $\Lambda$ vacuum energy, and in doing so keep the bits, for computational bits cosmological evolution even if $R_{\text {initial }} \rightarrow 0$. We state that the presence of computational bits is necessary for cosmological evolution to commence.
\end{abstract}

\section{Keywords}

Fjortoft Theorem, Thermodynamic Potential, Matter Creation, Vacuum Energy Non-Pathological Singularity Affecting Einstein Equations, Planck Length, Braneworlds

\section{Introduction}

This article is to investigate what happens physically if there is a non-pathological singularity in terms of Eins- 
teins equations at the start of space-time. This eliminates the necessity of having then put in the Planck length since then they would be no reason to have a minimum non-zero length. The reasons for such a proposal come from [1] by Stoica who may have removed the reason for the development of Planck's length as a minimum safety net to remove what appears to be unadvoidable pathologies at the start of applying the Einstein equations at a space-time singularity, and are commented upon in this article. $\rho \sim H^{2} / G \Leftrightarrow H \approx a^{-1}$ in particular is remarked upon. This is a counter part to Fjortoft theorem in Appendix I. The idea is that entanglement entropy will help generate bits, due to the presence of a vacuum energy, as derived at the end of the article, and the presence of a vacuum energy non-zero value, is necessary for comsological evolution. Before we get to that creation of what is a necessary creation of vacuum energy conditions, we refer to constructions leading to extremely pathological problems which [1] leads to minus the presence of initial non-zero vacuum energy. [2] also adds more elaboration on this.

Note a change in entropy formula given by Lee [3] about the inter relationship between energy, entropy and temperature as given by

$$
m \cdot c^{2}=\Delta E=T_{U} \cdot \Delta S=\frac{\hbar \cdot a}{2 \pi \cdot c \cdot k_{B}} \cdot \Delta S
$$

As a reviewer has asked about Equation (1) and the inter relationship of a mass $m$, and acceleration, the key point of this review is to look at if gravitons have a mass, $m$, in the beginning, and if Equation (1) is used, which the mass of a graviton is proportional to the following

$$
m=\frac{\Delta E}{c^{2}}=\frac{T_{U} \cdot \Delta S}{c^{2}}=\frac{\hbar \cdot a}{2 \pi \cdot c^{4} \cdot k_{B}} \cdot \Delta S
$$

The reason why the mass of a graviton is stated as given by Equation (1a) is to presume that the relationship given by Lee [3], as to any mass, is given by Equation (1) and Equation (1a), so we can relate any presumed mass linked to gravitons to change in entropy. As to acceleration appearing, the acceleration, $a \cong \frac{c^{2}}{\Delta x}$ is part of the formula given by Equation (1) and by default Equation (1a) and also by thermodynamic reasoning the generalized temperature

$$
T_{U}=\frac{\hbar \cdot a}{2 \pi \cdot c^{2} \cdot k_{B}}
$$

If we assume, in the onset of expansion of the universe, that Equation (1b) holds, then we can review the application of Equation (1a) to graviton mass, $m$, as $m=\frac{\Delta E}{c^{2}}=\frac{T_{U} \cdot \Delta S}{c^{2}}$, and to have acceleration, given by $a \cong \frac{c^{2}}{\Delta x}$ as part of a definition of generalized temperature, given by Equation (1b).

Note that temperature is, in this presentation by Lee [3] presumably a constant initially, i.e. very hot, so then we are really in this presentation, assuming that the acceleration as given by $a \cong \frac{c^{2}}{\Delta x}$ is a constant, so in fact what we are actually reviewing through Equation (1a) is a direct relationship of mass as proportional to entropy, i.e. as

$$
m \sim \Delta S
$$

i.e. the mass of a graviton is presumed to be proportional to entropy, i.e. in choosing Equation (1c) we are leading up to one of the themes of this document which is that if entropy is proportional to information and note that later, we will be relating entropy, as given, to a numerical count factor, i.e. then in fact, this will lead to a re write of Equation (1c) to read as, if $N$ (count) is a numerical count proportional to the change in Entropy, that

$$
m \sim \Delta S \sim N(\text { count }) \Rightarrow m_{\text {graviton }} \sim \frac{\Delta S}{N(\text { count })}
$$

This assumes that we are evaluating Equation (1b) as a constant, i.e. that the temperature be fixed, which is 
leading to the acceleration, which the referee was so concerned about, as a constant, i.e. via the relationship of looking at $a \cong \frac{c^{2}}{\Delta x}$ as an acceleration factor, and presumably that the delta $\mathrm{x}$ factor in acceleration is of the interval of Planck length.

Lee's formula is crucial for what we will bring up in the latter part of this document. Namely that changes in initial energy could effectively vanish if [1] is right, i.e. Stoica removing the non pathological nature of a big bang singularity. That is, unless entanglement entropy is used.

If the mass $m$, i.e. for gravitons is set by acceleration (of the net universe) and a change in enthropy $\Delta S \sim 10^{38}$ between the electroweak regime and the final entropy value of, if $a \cong \frac{c^{2}}{\Delta x}$ for acceleration is used, so then we obtain

$$
S_{\text {Today }} \sim 10^{88}
$$

Then we are really forced to look at (1) as a paring between gravitons (today) and gravitinos (electro weak) in the sense of preservation of information.

Having said this note by extention $\rho \sim H^{2} / G \Leftrightarrow H \approx a^{-1}$. As $\rho$ changes due to $\rho \sim H^{2} / G$ and $R_{\text {initial }} \sim \frac{1}{\#} \ell_{\mathrm{Ng}}<l_{\text {Planck }}, t$ hen $a$ is also altered i.e. goes to zero.

What will determine the answer to this question is if $\Delta E_{\text {initial }}$ goes to zero if $R_{\text {initial }} \rightarrow 0$ which happens if there is no minimum distance mandated to avoid the pathology of singularity behavior at the heart of the Einstein equations. In doing this, we avoid using the energy $E \rightarrow 0^{+}$situation, i.e. of vanishing initial space-time energy, and instead refer to a nonzero energy, with $\Delta E_{\text {initial }}$ instead vanishing. In particular, the Entanglement entropy concept as presented by Muller and Lousto [4] is presented toward the end of this manuscript as a partial resolution of some of the pathologies brought up in this article before the entanglement entropy section. No matter how small the length gets, $S_{\text {entropy }}$ if it is entanglement entropy, will not go to zero. The requirement is that the smallest length of time, $t$, rescaled, does not go to zero. This preserves a minimum non-zero $\Lambda$ vacuum energy, and in doing so keep non zero amounts of initial bits, for computational bits cosmological evolution even if $R_{\text {initial }} \rightarrow 0$.

I think that the common confusion here, is that $R_{\text {initial }} \rightarrow 0$ refers to initial RADII and not to curvature, which was also one of the questions raised by the referee. $R_{\text {initial }} \sim \frac{1}{\#} \ell_{\mathrm{Ng}}<l_{\text {Planck }}$ is a minimum radii and has nothing to do with curvature. This formula, which evidently confused referees, i.e. if \# refers to a computational bits value which will show up in our manuscript, then our statement is that we have an initial radii of less than Planck Length. As given by

$$
R_{\text {initial }} \sim \frac{1}{\#} \ell_{N g}<l_{\text {Planck }}
$$

Is part of the build up of Equation (3) and should be read by readers so as to understand the significance of what is in this Equation (2a). I.e. $l_{\mathrm{Ng}}<l_{\text {Planck }}$ does not hold, in general, and we get Equation (2a) only if the \# value is used which refers to a computational bits value.

Before doing that, we review $\mathrm{Ng}$ [5] and his quantum foam hypothesis to give conceptual underpinnings as to why we later even review the implications of entanglement Entropy.

We state unequivocally here, that Equation (2a) has \# referring to a computational bits value which is Equation (3) and will be part of treating entropy and its evolution

Note that this evaluation is preformed in the Planck time interval, and is the basis of evaluation by our paper.

I.e. the concept of bits and computations is brought up because of applying energy uncertainty, as given by [5] and the Margolis theorem appears to indicate that the universe could not possibly evolve if [1] is applied, in a 4 dimensional closed universe. This bottle neck as indicated by Ng's [5] formalism is even more striking in the author's end of article proof of the necessity of using entanglement entropy in lieu of the conclusion involving entanglement entropy, which can be non zero, even if $R_{\text {initial }} \rightarrow 0$ provided there is a minimum non-zero time length. 


\section{Review of $\mathrm{Ng}$, [5] with Comments}

First of all, Ng refers to the Margolus-Levitin theorem with the rate of operations $<E / \hbar$ $\Rightarrow$ \# operations $<E / \hbar \times$ time $=\frac{M c^{2}}{\hbar} \cdot \frac{l}{c}$. Ng wishes to avoid black-hole formation $\Rightarrow M \leq \frac{l c^{2}}{G}$. This last step is not important to our view point, but we refer to it to keep fidelity to what Ng brought up in his presentation. Later on, $\mathrm{Ng}$ refers to the \# operations $\leq\left(R_{H} / l_{P}\right)^{2} \sim 10^{123}$ with $R_{H}$ the Hubble radius. Next Ng refers to the \#bits $\propto[\text { \# operations] }]^{3 / 4}$. Each bit energy is $1 / R_{H}$ with $R_{H} \sim l_{P} \cdot 10^{123 / 2}$

The key point as seen by $\mathrm{Ng}[4]$ and the author is in

$$
\text { \#bits } \sim\left[\frac{E}{\hbar} \cdot \frac{l}{c}\right]^{3 / 4} \approx\left[\frac{M c^{2}}{\hbar} \cdot \frac{l}{c}\right]^{3 / 4}
$$

Assuming that the initial energy $\mathrm{E}$ of the universe is not set equal to zero, which the author views as impossible, the above equation says that the number of available bits goes down dramatically if one sets

$R_{\text {initial }} \sim \frac{1}{\#} \ell_{\mathrm{Ng}}<l_{\text {Planck }}$ ? Also Ng writes entropy $S$ as proportional to a particle count via $N$.

$$
S \sim N \cong\left[R_{H} / l_{P}\right]^{2}
$$

We rescale $R_{H}$ to be

$$
\left.R_{H}\right|_{\text {rescale }} \sim \frac{l_{\mathrm{Ng}}}{\#} \cdot 10^{123 / 2}
$$

The upshot is that the entropy, in terms of the number of available particles drops dramatically if \# becomes larger.

So, as $R_{\text {initial }} \sim \frac{1}{\#} \ell_{\mathrm{Ng}}<l_{\text {Planck }}$ grows smaller, as \# becomes larger.

a) The initial entropy drops;

b) The nunber of bits initially available also drops.

The limiting case of (4) and (5) in a closed universe, with no higher dimensional embedding is that both would almost vanish, i.e. appear to go to zero if \# becomes very much larger. The quest4ion we have to ask is would the number of bits in computational evolution actually vanish?

\section{Does It Make Sense to Talk of Vacuum Energy If $R_{\text {initial }} \neq 0$ Is Changed to $R_{\text {initial }} \rightarrow 0$ ? Only Answerable Straightforwardly If an Embedding Superstructure Is Assigned. Otherwise Difficult, Unless One Is Using Entanglement Entropy Which Is Non Zero Even If $R_{\text {initial }} \rightarrow 0$}

We summarize what may be the high lights of this inquiry leading to the present paper as follows:

a. One could have the situation if $R_{\text {initial }} \rightarrow 0$ of an infinite point mass, if there is an initial nonzero energy in the case of four dimensions and no higher dimensional embedding even if [1] goes through verbatim. The author sees this as unlikely. The infinite point mass construction is verbatim if one assumes a closed universe, with no embedding superstructure and no entanglement entropy. Note this appears to nullify the parallel Brane world construction used by Durrer [6]. The author, in lieu of the manuscript sees no reason as to what would perturb this infinite point structure, so as to be able to enter in a big bang era. In such a situation, one would not have vacuum energy unless entanglement entropy were used. That is unless one has a non zero entanglement entropy [4] present even if $R_{\text {initial }} \rightarrow 0$. See [7] for a smilar argument.

b. The most problematic scenario. $R_{\text {initial }} \rightarrow 0$ and no initial cosmological energy. i.e. this in a 4 dimensional closed universe. Then there would be no vacuum energy at all. initially. A literal completely empty initial state, which is not held to be viable by Volovik [8].

c. If additional dimensions are involved in beginning cosmology, than just 4 dimensions will lead to physics which may give credence to other senarios. One scenario being the authors speculation as to initial degrees of 
freedom reaching up to 1000, and the nature of a phase transition from essentially very low degrees of freedom, to over 1000 as speculated by the author in 2010 [9].

d. What the author would be particularly interested in knowing would be if actual semiclassical reasoning could be used to get to an initial prequantum cosmological state. This would be akin to using [10], but even more to the point, using [11] and [12], with both these last references relevant to forming Planck's constant from electromagnetic wave equations. The author points to the enormous electromagnetic fields in the electroweak era as perhaps being part of the background necessary for such a semiclassical derivation, plus a possible octonionic space-time regime, as before inflation flattens space-time, as forming a boundary condition for such constructions to occur [13].

The relevant template for examinging such questions is given in Table 1 as printed.

e. The meaning of octonionic geometry prior to the introduction of quantum physics presupposes a form of embedding geometry and in many ways is similar to Penrose's cyclic conformal cosmology speculation.

f. It is striking how a semiclassical argument can be used to construct in Table 1. In particular, we look at how Planck's constant is derived, as in the electroweak regime of space-time, for a total derivative [11] [12]

$$
E_{y}=\frac{\partial A_{y}}{\partial t}=\omega \cdot A_{y}^{\prime}(\omega \cdot(t-x))
$$

Similarly [11] [12]

$$
B_{z}=-\frac{\partial A_{y}}{\partial x}=\omega \cdot A_{y}^{\prime}(\omega \cdot(t-x))
$$

The $A$ field so given would be part of the Maxwell's equations given by [10] as, when [ ] represents a D'Albertain operator, that in a vacuum, one would have for an $A$ field [11] [12]

$$
[] A=0
$$

And for a scalar field $\phi$

$$
[] \phi=0
$$

Following this line of thought we then would have an energy density given by, if $\varepsilon_{0}$ is the early universe permeability [11]

$$
\eta=\frac{\varepsilon_{0}}{2} \cdot\left(E_{y}^{2}+B_{z}^{2}\right)=\omega^{2} \cdot \varepsilon_{0} \cdot A_{y}^{\prime 2}(\omega \cdot(t-x))
$$

We integrate (10) over a specified $E$ and $M$ boundary, so that, then we can write the following condition namely [11] [12].

$$
\iiint \eta \mathrm{d}(t-x) \mathrm{d} y \mathrm{~d} z=\omega \varepsilon_{o} \iiint A_{y}^{\prime 2}(\omega \cdot(t-x)) \mathrm{d}(t-x) \mathrm{d} y \mathrm{~d} z
$$

(11) would be integrated over the boundary regime from the transition from the octonionic regime of space time, to the non-octonionic regime, assuming an abrupt transition occurs, and we can write, the volume integral as representing [11] [12]

$$
E_{\text {gravitational-energy }}=\hbar \cdot \omega
$$

Then by applying [11] [12] we get $\hbar$ formed by semiclassical reasons. In semiclassical reasoning similar to [10].

\section{Table 1. Time interval dynamical consequences does QM/WdW apply?}

Just before electroweak era

Electro-Weak Era

Post Electro-Weak Era to today
Form $\hbar$ from early $E$ \& $M$ fields, and use Maxwell's

Equations with necessary to implement boundary conditions created from change from octonionic geometry to flat space
NO

YES

YES Wave function of Universe 


$$
\hbar(t) \underset{\text { Apply-Machs-Relations }}{\longrightarrow} \hbar \text { (Constant value) }
$$

The question we can ask, is that can we have a prequantum regime commencing for (11) and (12) for $\hbar$ if $R_{\text {initial }} \sim \frac{1}{\#} \ell_{\mathrm{Ng}}<l_{\text {Planck }}$ ? And a closed 4 dimensional universe? If so, then what is the necessary geometrial regime of space-time so that the integration performed in (11) can commence properly? Also, what can we say about the formation of (12) above, as a number, \# gets larger and larger, effectively leading to. Also, with an octonionic geometry regime which is a pre quantum state [13].

In so many words, the formation period for $\hbar$ is our pre-quantum regime. Table 1 could even hold if $R_{\text {initial }} \rightarrow 0$ but that the 4 dimensional space-time exhibiting such behavior is embedded in a higher dimensional template. That due to $R_{\text {initial }} \rightarrow 0$ not removing entanglement entropy as is discussed near the end of this article.

\section{If $R_{\text {initial }} \rightarrow 0$ Then If There Is an Isolated, Closed Universe, There Is a Disaster Unless One Uses Entanglement Entropy}

One does not have initial entropy, and the number of bits initially disappears. That is if one is not using entanglement entropy, as will be examined at the end of this article.

Abandoning the idea of a completely empty universe, this unperturbed point of space time (of matter-energy) appears to be a configuration for a static point of space time with no perturbation, as may be the end result of applying Fjortoft theorem [14] to the thermodynamic potential as given in [15], i.e. the non definitive answer for fulfillment of criteria of instability by applying Fjortoft's theorem [14] to the potential [15] leading to no instability as given by the potential given in [15] may lead to a point of space-time with no change, i.e. a singular point with "infinite" mass which does not change at all. This issue will be reviewed in [16] a different procedure, i.e. a so called nonsingular universe construction. To get there we will first of all review an issue leading up to implementation of [16].

\section{Can an Alternative to a Minimum Length Be Put in? Consider the Example of Planck Time as the Minimal Component, Not Planck Length}

From J. Dickau, [17] the following was given to the author, as a counter point to $R_{\text {initial }} \rightarrow 0$ leading to a disaster.

"If we examine the Mandelbrot Set along the Real axis, it informs us about behaviors that also pertain in the quaternion and octonic case-because the real axis is invariant over the number types. If numbers larger than.25 are squared and summed recursively (i.e. $-z=z^{\wedge} 2+c$ ) the result will blow up, but numbers below this threshold never get to infinity, no matter how many times they are iterated. But once space-like dimensions are added-i.e. an imaginary compoent - the equation blows up exponentially, faster than when iterated.

Dickau concludes:

"Anyhow there may be a minimum (space-time length) involved but it is probably in the time direction".

This is a counter pose to the idea of minimum length, looking at a beginning situation with a crucial parameter $R_{\text {initial }}$ even if the initial time step is “put in by hand”. First of all, look at [4], if $E$ is $M$, due to setting $c=1$, then

$$
\Delta E_{\text {initial }} \approx 4 \pi \rho\left(R_{\text {initial }}\right)^{2} \Delta R_{\text {initial }}
$$

Everything depends upon the parameter $R_{\text {initial }}$ which can go to zero. We have to look at what (14) tells us, even if we have an initial time step for which time is initially indeterminate, as given by a redoing of Mitra's $g_{00}$ formula [7] which we put in to establish the indeterminacy of the initial time step if quantum processes hold.

$$
\left(g_{00}=\exp \left[\frac{-2}{1+(\rho(t) / p(t))}\right]\right) \underset{\rho+p=0}{\longrightarrow} 0
$$

What Dickau is promoting is, that the Mandelbrot set, if applicable to early universe geometry, that what the author wrote, with $R_{\text {initial }} \sim \frac{1}{\#} \ell_{\mathrm{Ng}}<l_{\text {Planck }} \underset{\# \neq \infty}{\longrightarrow}$ small-value potentially going to zero, is less important than a 
minimum time length. The instability issue is reviewed in Appendix II for those who are interested in the author's views as to lack proof of instability. It uses [15] which the author views as THE reference as far as thermodynamic potentials and the early universe.

\section{Muller and Lousto Early Universe Entanglement Entropy, and Its Implications: Solving the Spatial Length Issue, Provided a Minimum Time Step Is Preserved in the Cosmos, in Line with Dickau's Suggestion}

We look at [4]

$$
S_{\text {Entropy }}=0.3 r_{H}^{2} / a^{2} \text { for a time dependent horizon radius } r_{H} \text { in cosmology }
$$

Equation (16) above was shown by the author to be fully equivalent to

$$
S_{\text {Entropy }}=0.3 r_{H}^{2} / a^{2} \sim \frac{0.3}{a^{2}} \exp \left[-t \cdot \sqrt{\frac{\Lambda}{3}}\right]
$$

i.e.

$$
\left[-t \sqrt{\frac{\Lambda}{3}}\right] \sim \ln _{e}\left(\frac{a^{2}}{0.3} \cdot S_{\text {entropy }}\right)
$$

So, then one has

$$
\Lambda \approx \frac{3}{t^{2}} \cdot\left[\ln _{e}\left(\frac{a^{2}}{0.3} \cdot S_{\text {entropy }}\right)\right]^{2}
$$

No matter how small the length gets, $S_{\text {entropy }}$ if it is entanglement entropy, will not go to zero. The requirement is that the smallest length of time, $\mathrm{t}$, re scaled does not go to zero. This preserves a minimum non zero $\Lambda$ vacuum energy, and in doing so keep the non zero initial bits, for computational bits contributions to evolving space time behavior even if $R_{\text {initial }} \rightarrow 0$.

\section{Reviewing a Suggestion as to How to Quantify the Shrinkage of the Scale Factor and Its Connections with Entanglement Entropy}

We are given by [16] if there is a non singular universe, a template as to how to evaluate scale factor $a$ against time scaled over Planck time, with the following results.

$$
\ln _{e} a+\frac{a^{6}}{6}+\frac{2 \cdot a^{3}}{3}=\sqrt{\frac{8 \pi}{3}} \cdot \frac{t}{t_{\text {Planck }}}
$$

Two time and scale factor values in tandem particularly stand out. Namely,

$$
a \sim \frac{a_{\text {scale }}}{\left[a_{\text {Planck }} \sim 10^{-25}\right]} \equiv 1.344 \Leftrightarrow t \propto t_{\text {Planck }} \sim 5.4 \times 10^{-44} \mathrm{sec}
$$

Also

$$
a \sim \frac{a_{\text {scale }}}{\left[a_{\text {Planck }} \sim 10^{-25}\right]} \equiv 0.7414 \Leftrightarrow t \propto 0^{+}
$$

The main thing we can take from this, is to look at the inter-relationship of how to pin down an actual initial Hubble "constant" expansion parameter, where we look at:

$$
1.813=\exp \left(H_{\text {Planck }} \cdot t_{\text {Planck }}\right) \Leftrightarrow H_{\text {Planck }}=\frac{\ln _{e}(1.813)}{t_{\text {Planck }}}
$$

Recall that $\Lambda \approx \frac{3}{t^{2}} \cdot\left[\ln \left(\frac{a^{2}}{0.3} \cdot S_{\text {entropy }}\right)\right]^{2}$, which is predicated upon, if the time is close to Planck time the ini- 
tial maximal density of

$$
\rho_{\text {Planck }} \sim 5.2 \times 10^{96} \mathrm{~kg} / \mathrm{m}^{3}
$$

And length given by

$$
\text { Length }(\text { Planck })=l_{\text {Planck }} \sim 1.6 \times 10^{-35} \text { meters }
$$

So (24) is implying that the amount of matter in a region of space $\left(l_{\text {Planck }}\right)^{3}$ is initially about

$$
\rho_{\text {initial }} \sim 2 \times 10^{-10} \mathrm{~kg} \sim 2 \times 10^{-7} \text { grams }
$$

Using $1 \mathrm{GeV} / \mathrm{c}^{2}=1.783 \times 10^{-27} \mathrm{~kg}$ means that (26) above is

$$
\rho_{\text {initial }} \sim 2 \times 10^{-10} \mathrm{~kg} \sim 2 \times 10^{-7} \text { grams } \sim 10^{+17} \mathrm{GeV}
$$

Then if

$$
10^{+17} \mathrm{GeV} \sim \Lambda \approx \frac{3}{t^{2}} \cdot\left[\ln \left(\frac{a^{2}}{0.3} \cdot S_{\text {entropy }}\right)\right]^{2}
$$

It will lead to

$$
10^{+17} \mathrm{GeV} \times 10^{-70} \sec ^{2} \sim\left[\ln e\left(\frac{a^{2}}{0.3} \cdot S_{\text {entropy }}\right)\right]
$$

Then, to first order, one is looking at initial entropy to get a non zero but definite vacuum energy as leading to an entanglement entropy of about (just before the electro weak regime) regardless of the situation being in fidelity , or lack of with the physics of [18]

$$
S_{\text {entropy }} \sim 1 / a^{2} \sim 10^{20}-10^{40}
$$

\section{Reviewing the Geometry for Embedding (29) above}

In line with Stoica [1] shrinking the minimum length and referring to both (29) and (27), the idea is to use a surface area treatment as to getting the initial entropy values as given in (29). To do so, the author at the situation presented in Figure 1.

The two branes presented in Figure 1 given at $y_{b}$ and $y_{s}$ refer to the two Brane world states, especially in line with [19] [20]. The first one, namely $y_{b}$ is the Brane where our physical universe lives in, and is embedded in. If one uses this construction, with higher dimensions than just 4 dimensions, then it is possible to have a single point in 4 dimensional space as a starting point to a tangential sheet which is part of an embedding in more than 4 dimensions. Along the lines of having a 4 dimensional cusp with its valley (lowest) point in a more than 4 dimensional tangential surface. The second Brane is about $10^{-30}$ centimeters away from the Brane our physical world lives in, and moves closer to our own Brane in the future, leading to a slapping of the two Branes together about a trillion years ahead in our future [19] [20]. The geometry we are referring to with regards to embedding is in the first Brane $y_{b}$. [6] uses this geometry to have graviton production which the author has used to model Dark Energy.

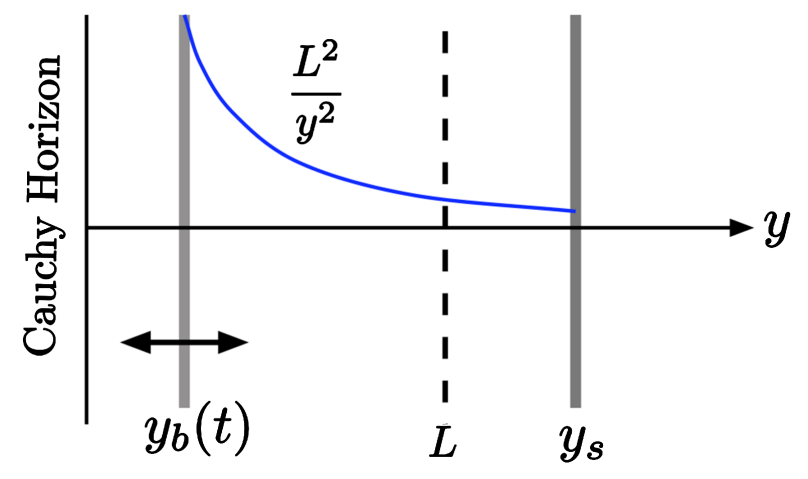

Figure 1. As adopted from Reference [6]. 


\section{Conclusion: Making Computational Bits, via (19)}

As stated by Ng. the idea would be to have to give imputs into (3) i.e.

$$
\text { \#bits } \sim\left[\frac{E}{\hbar} \cdot \frac{l}{c}\right]^{3 / 4} \approx\left[\frac{M c^{2}}{\hbar} \cdot \frac{l}{c}\right]^{3 / 4}
$$

Here in this case, even if the spatial contribution, due to [1] goes to zero, the idea would be to have the time length non-zero so as to have a space-time version of $l$ non-zero. This would also be in tandem with calling $E$, in (3) as proportional to $\Lambda \approx \frac{3}{t^{2}} \cdot\left[\ln _{e}\left(\frac{a^{2}}{0.3} \cdot S_{\text {entropy }}\right)\right]^{2}$, where if the time is Planck time, in minimum value, and $S_{\text {entropy }} \sim 1 / a^{2} \sim 10^{20}-10^{40}$ in value, one would have before the electro-weak an input into $E$, which would require an entropy (entanglement).

What remains to be seen is, if there is a geometric sheet in more than 4 dimensions, allowing for non-zero time, as argued for $\Lambda \approx \frac{3}{t^{2}} \cdot\left[\ln \left(\frac{a^{2}}{0.3} \cdot S_{\text {entropy }}\right)\right]^{2}$, even if the spatial component goes to zero, according to [1]. We suggest an update as to what was written by Seth Lloyd [21] with

$$
I=S_{\text {total }} / k_{B} \ln 2=[\# \text { operations }]^{3 / 4}=\left[\rho \cdot c^{5} \cdot t^{4} / \hbar\right]^{3 / 4}
$$

when [22]

$$
\rho \equiv T^{00} \sim \Lambda_{\text {vacuum-energy }}
$$

While doing this, a good thing to do, would be to keep in mind the four dimensional version of vacuum energy as given by Park, [23] namely

$$
\Lambda_{4-\operatorname{dim}} \approx c_{2} \cdot T^{\beta}
$$

As well as the transition given by a combination of [23], with [24], Barvinskey et al.

$$
\Lambda_{4-\operatorname{dim}} \propto c_{2} \cdot T \underset{\text { graviton-production }}{\longrightarrow} 360 \cdot m_{P}^{2} \ll c_{2} \cdot\left[T \approx 10^{32} \mathrm{~K}\right]
$$

Quantifying the above, and giving it experimental proof, via detector technology may allow us to investigate an old suggestion by the author as to four dimension and five dimensional vacuum energy which are given for small time values $t \approx \delta^{1} \cdot t_{P}, \quad 0<\delta^{1} \leq 1$ and for temperatures sharply lower than $T \approx 10^{12}$ Kelvin, Beckwith [22] (2007), where for a positive integer $n$

$$
\frac{\Lambda_{4-\operatorname{dim}}}{\left|\Lambda_{5-\operatorname{dim}}\right|}-1 \approx \frac{1}{n}
$$

In particular, the author is interested in investigating if the following is true.

Look at an argument provided by Thanu Padmanabhan [25], leading to the observed cosmological constant value suggested by Park [22]. Assume that $l_{P} \sim 10^{-33} \mathrm{~cm} \underset{\text { Quantum-Gravity-threshold }}{\longrightarrow} \tilde{N}^{\alpha} \cdot l_{P}$, but that when we make this substitution that $1 \leq \tilde{N}^{\alpha} \leq 10^{2} \quad[26]$

$$
\rho_{V A C} \sim \frac{\Lambda_{\text {observed }}}{8 \pi G} \sim \sqrt{\rho_{U V} \cdot \rho_{I R}} \sim \sqrt{l_{\text {Planck }}^{-4} \cdot l_{H}^{-4}} \sim l_{\text {Planck }}^{-2} \cdot H_{\text {observed }}^{2}
$$

i.e. looking at if

$$
\Delta \rho \approx \text { a dark energy density } \sim H_{\text {observed }}^{2} / G
$$

Now to make it more interesting.

We can replace $\Lambda_{\text {observed }}, H_{\text {observed }}^{2}$ by $\Lambda_{\text {initial }}, H_{\text {initial }}^{2}$. In addition, we may look at inputs from the initial value 
of the Hubble parameter to get the necessary e folding needed for inflation, according to

$$
E \text {-foldings }=H_{\text {initial }} \cdot\left(t_{\text {End of inf }}-t_{\text {beginning of inf }}\right) \equiv N \geq 100 \Rightarrow H_{\text {initial }} \geq 10^{39}-10^{43}
$$

Leading to

$$
a(\text { End-of-inf }) / a(\text { Beginning-of-inf }) \equiv \exp (N)
$$

If we set $\Lambda_{\text {initial }} \sim C_{1} \cdot\left[T \sim 10^{32}\right.$ Kelvin $]$ implying a very large initial cosmological constant value, we get in line with what Park suggested for times much less than the Planck interval of time at the instant of nucleation of a vacuum state

$$
\Lambda_{\text {initial }} \sim\left[10^{156}\right] \cdot 8 \pi G \approx \text { huge number }
$$

Question. Do we always have this value of (39)? Can we say that Eq. (39) is such a large number at the onset of inflation? When we are not that far away from a volume of space characterized by $l_{p}^{3}$, or at most 100 or so times larger? Contemporary big bang theories imply this, i.e. a very high level of thermal energy. We need to ask if Eq. (29) with its enormous vacuum energy is due to vacuum energy being transferred from a prior universe, or if it is due to a pop up nucleation effect, i.e. emergent space time. This question is what should be investigated throughly. Appendix III and Appendix IV give suggestions which the author has thought of which may contribute to, if anything, models of how instantons from a prior universe may be transmitted to our present universe, i.e. Appendix $\mathbf{V}$ which is based in part on what Wesson formulated as to five dimensional universe constructions, and instantons [27]. The very interesting topic of vacuum fluctuations in such space-time has also been reviewed briefly in Appendix VI, and Appendix VII. Appendix VIII which is taken directly from [28] concludes with references to the work by Corda as to different models of gravity, and to work as to the Ligo collaboration, which should be met by any model of Equation (39) which passes experimental muster, i.e. the objections and technical points raised in Appendix VIII will be of decisive import as to satisfying [28]-[35] as far as the existence of LIGO instruments and other forms of GW tests which recently have borne fruit as to the detection of GW [36]. In particular, Equation (39) and what number we pick for the initial vacuum state has to produce results which are in fidelity with the requirements of reference [31], and furthermore will have to take into consideration [36] as well.

\section{Acknowledgements}

This work is supported in part by National Nature Science Foundation of China grant No. 110752.

\section{References}

[1] Stoica, C. (2012) Beyond the FRWL Big Bang Singularity. http://arxiv.org/pdf/1203.1819.pdf

[2] Beckwith, A.W. (2012) Is Quantum Mechanics Involved at the Start of Cosmological Evolution? Does a Machian Relationship between Gravitons and Gravitinos Answer This Question? http://vixra.org/abs/1206.0023

[3] Lee, J.-W. (2012) On the Origin of Entropic Gravity and Inertia. Foundations of Physics, 42. http://arxiv.org/abs/1003.4464;

[4] Muller, R. and Lousto, A.C. (1995) Entanglement Entropy in Curved Space-Times with Event Horizons. arXIV gr-qc/9504049v1.

[5] Ng, Y.J. and van Dam, H. (2000) Spacetime Foam: From Entropy and Holography to Infinite Statistics and Nonlocality. Foundations of Physics, 30, 795-805.

[6] Ruser, M. and Durrer, R. (2007) Dynamical Casimir Effect for Gravitons in Bouncing Braneworlds. Physical Review D, 76, Article ID: 104014. http://arxiv.org/abs/0704.0790

[7] Mitra, A. (2011) Why the Big Bang Model Cannot Describe the Observed Universe Having Pressure and Radiation. Journal of Modern Physics, 2, 1436-1442.

[8] Volovik, G.E. (2006) Vacuum Energy: Myths and Realities. arXIV: gr-qc/0604062 v2.

[9] Beckwith, A. (2011) How to Use the Cosmological Schwinger Principle for Energy Flux, Entropy, and “Atoms of Space-Time” to Create a Thermodynamic Space-Time and Multiverse. 5th International Workshop DICE2010: Space-Time-Matter-Current Issues in Quantum Mechanics and Beyond. http://iopscience.iop.org/1742-6596/306/1;jsessionid=A05372A78C18D970BF35F40A9A863B51.c2 
http://iopscience.iop.org/1742-6596/306/1/012064

[10] Gryzinski, M. (1959) Classical Theory of Electronic and Ionic Inelastic Collisions. Physical Review, 115, 374-383. http://dx.doi.org/10.1103/PhysRev.115.374

[11] Bruchholz, U. (2009) Derivation of Planck’s Constant from Maxwell’s Electrodynamics. Progress in Physics, 4, 67. http://www.ptep-online.com/index_files/2009/PP-19-07.PDF

[12] Bruzchholz, U. (2009) Key Notes on a Geometric Theory of Fields. Progress in Physics, 2, 107-113.

[13] Beckwith, A. (2011) Octonionic Gravity Formation, Its Connections to Micro Physics. Open Journal of Microphysics, 1, 13-18. http://dx.doi.org/10.4236/ojm.2011.11002

[14] Pringle, J. and King, A. (2007) Astrophysical Flows. Cambridge University Press, New York. http://dx.doi.org/10.1017/CBO9780511802201

[15] Padmanabhan, T. (2011) Lessons from Classical Gravity about the Quantum Structure of Spacetime. Journal of Physics: Conference Series, 306, Article ID: 012001. http://iopscience.iop.org/1742-6596/306/1/012001 http://dx.doi.org/10.1017/CBO9780511802201

[16] Gao, C. (2012) A Model of Nonsingular Unvierse. Entropy, 14, 1296-1305. http://dx.doi.org/10.3390/e14071296

[17] Dickau, J. (2012) Private Communication to the Author as to Minimum Space-Time "Length" provided as of 7 August 212.

[18] Surya, S. (2010) In Search of a Covariant Quantum Measure. Journal of Physics: Conference Series, 306, Article ID: 012018. http://iopscience.iop.org/1742-6596/306/1/012018 http://dx.doi.org/10.1088/1742-6596/306/1/012018

[19] Koury, J., Steinhardt, P.J. and Turkok, N. (2004) Designing Cyclic Universe Models. Physical Review Letters, 92, Article ID: 031302. http://dx.doi.org/10.1103/PhysRevLett.92.031302

[20] Koury, J., Steinhardt, P.J. and Turkok, N. (2003) Inflation versus Cyclic Predictions for Spectral Tilt. Physical Review Letters, 91, Article ID: 161301. http://dx.doi.org/10.1103/PhysRevLett.91.161301

[21] Lloyd, S. (2002) Computational Capacity of the Universe. Physical Review Letters, 88, Article ID: 237901. http://dx.doi.org/10.1103/PhysRevLett.88.237901

[22] Beckwith, A.W. (2007) Symmetries in Evolving Spacetime from Prior to Present Universes. arXIV math-ph/0501028

[23] Park, D.K., Kim, H. and Tamarayan, S. (2002) Nonvanishing Cosmological Constant of Flat Universe in Brane World Senarios. Physics Letters B, 535, 5-10. http://dx.doi.org/10.1016/S0370-2693(02)01729-X

[24] Barvinsky, A., Kamenschick, A. and Yu, A. (2006) Thermodynamics from Nothing: Limiting the Cosmological Constant Landscape. Physical Review D, 74, Article ID: 121502. (Rapid communications) http://dx.doi.org/10.1103/PhysRevD.74.121502

[25] Padmanabhan, T. (2006) An Invitation to Astro Physics. World Scientific Publishing Co. Pte. Ltd, Singapore.

[26] Padmanabhan, T. (2005) Understanding Our Universe: Current Status and Open Issues. In: Ashtekar, A., Ed., 100 Years of Relativity Space-Time Structure: Einstein and Beyond, World Press Scientific, Singapore, 175-204.

[27] Roger, P. (2010) Cycles of Time: An Extrardinary New View of the Universe. The Bodley Head, the Random House Group, Ltd., London.

[28] Beckwith, A. (2016) Does a Randall-Sundrum Brane World Effective Potential Influence Axion Walls Helping to Form a Cosmological Constant Affecting Inflation? Journal of High Energy Physics, Gravitation and Cosmology, 2, 125-153. http://dx.doi.org/10.4236/jhepgc.2016.21013

[29] Beckwith, A. (2016) Non Linear Electrodynamics Contributing to a Minimum Vacuum Energy (“Cosmological Constant”) Allowed in Early Universe Cosmology. Journal of High Energy Physics, Gravitation and Cosmology, 2, 25-32. http://dx.doi.org/10.4236/jhepgc.2016.21003

[30] Corda, C. (2009) Interferometric Detection of Gravitational Waves: The Definitive Test for General Relativity. International Journal of Modern Physics D, 18, 2275-2282. http://arxiv.org/abs/0905.2502 http://dx.doi.org/10.1142/s0218271809015904

[31] Van Den Broeck, C., et al. (2015) Gravitational Wave Searches with Advanced LIGO and Advanced Virgo. http://arxiv.org/pdf/1505.04621v1.pdf

[32] Cowen, R. (2015) Gravitational Waves Discovery Now Officially Dead; Combined Data from South Pole Experiment BICEP2 and Planck Probe Point to Galactic Dust as Confounding Signal. http://www.nature.com/news/gravitational-waves-discovery-now-officially-dead-1.16830

[33] Cowen, R. (2014) Full-Galaxy Dust Map Muddles Search for Gravitational Waves. http://www.nature.com/news/full-galaxy-dust-map-muddles-search-for-gravitational-waves-1.15975

[34] Beckwith, A. (2016) Gedanken Experiment for Fluctuation of Mass of a Graviton, Based on the Trace of GR Stress 
Energy Tensor-Pre Planckian Conditions That Lead to Gaining of Graviton Mass, and Planckian Conditions That Lead to Graviton Mass Shrinking to $10^{-62}$ Grams. Journal of High Energy Physics, Gravitation and Cosmology, 2, 19-24. http://dx.doi.org/10.4236/jhepgc.2016.21002

[35] Beckwith, A. (2016) Gedanken Experiment for Degree of Flatness, or Lack of, in Early Universe Conditions. Journal of High Energy Physics, Gravitation and Cosmology, 2, 57-65. http://dx.doi.org/10.4236/jhepgc.2016.21006

[36] Abbott, B.P., et al. (LIGO Scientific Collaboration and Virgo Collaboration) (2016) Observation of Gravitational Waves from a Binary Black Hole Merger. Physical Review Letters, 116, Article ID: 061102. http://dx.doi.org/10.1103/PhysRevLett.116.061102

[37] Beckwith, A.W. (2011) Identifying a Kaluza Klein Treatment of a Graviton Permitting a Deceleration Parameter Q (Z) as an Alternative to Standard DE. Journal of Cosmology, 13. http://journalofcosmology.com/BeckwithGraviton.pdf

[38] Crowell, L. (2005) Quantum Fluctuations of Space-Time. World Scientific Series in Contemporary Chemical Physics, Vol. 25, World Scientific, Singapore.

[39] Misner, C., Thorne, K. and Wheeler, J. (1973) Gravitation. W.H. Freeman, and Company, San Francisco.

[40] Wesson, P. (1999) Space-Time-Matter, Modern Kaluza Klein Theory. World Scientific, Singapore.

[41] Samtleben, D., Staggs, S. and Winstein, B. (2007) The Cosmic Microwave Background for Pedestrians: A Review for Particle and Nuclear Physicists. Annual Review of Nuclear and Particle Science, 57, 245-283. 


\section{Appendix I. Fjortoft Theorem}

A necessary condition for instability is that if $z_{*}$ is a point in space time for which $\frac{\mathrm{d}^{2} U}{\mathrm{~d} z^{2}}=0$ for any given potential $U$, then there must be some value $z_{0}$ in the range $z_{1}<z_{0}<z_{2}$ such that

$$
\left.\frac{\mathrm{d}^{2} U}{\mathrm{~d} z^{2}}\right|_{z_{0}} \cdot\left[U\left(z_{0}\right)-U\left(z_{*}\right)\right]<0
$$

For the proof, see [12] and also consider that the main discussion is to find instability in a physical system which will be described by a given potential $U$. Next, we will construct in the boundary of the EW era, a way to come up with an optimal description for $U$.

\section{Appendix II. Constructing an Appropriate Potential for Using Fjortoft Theorem in Cosmology for the Early Universe Cannot Be Done. We Show Why}

To do this, we will look at Padamanabhan [15] and his construction of (in Dice 2010) of thermodynamic potentials he used to have another construction of the Einstein GR equations. To start, Padamanabhan [15] wrote

If $P_{c d}^{a b}$ is a so called Lovelock entropy tensor, and $T_{a b}$ a stress energy tensor

$$
\begin{aligned}
& U\left(\eta^{a}\right)=-4 \cdot P_{a b}^{c d} \nabla_{c} \eta^{a} \nabla_{d} \eta^{b}+T_{a b} \eta^{a} \eta^{b}+\lambda(x) g_{a b} \eta^{a} \eta^{b} \\
& =U_{\text {gravity }}\left(\eta^{a}\right)+U_{\text {matter }}\left(\eta^{a}\right)+\lambda(x) g_{a b} \eta^{a} \eta^{b} \\
& \Leftrightarrow U_{\text {matter }}\left(\eta^{a}\right)=T_{a b} \eta^{a} \eta^{b} ; U_{\text {gravity }}\left(\eta^{a}\right)=-4 \cdot P_{a b}^{c d} \nabla_{c} \eta^{a} \nabla_{d} \eta^{b}
\end{aligned}
$$

We now will look at

$$
\begin{gathered}
U_{\text {matter }}\left(\eta^{a}\right)=T_{a b} \eta^{a} \eta^{b} \\
U_{\text {gravity }}\left(\eta^{a}\right)=-4 \cdot P_{a b}^{c d} \nabla_{c} \eta^{a} \nabla_{d} \eta^{b}
\end{gathered}
$$

So happens that in terms of looking at the partial derivative of the top (1) equation, we are looking at

$$
\frac{\partial^{2} U}{\partial\left(\eta^{a}\right)^{2}}=T_{a a}+\lambda(x) g_{a a}
$$

Thus, we then will be looking at if there is a specified $\eta_{*}^{a}$ for which the following holds.

$$
\begin{aligned}
& {\left[\frac{\partial^{2} U}{\partial\left(\eta^{a}\right)^{2}}=T_{a a}+\lambda(x) g_{a a}\right]_{\eta_{0}^{a}} *\left[-4 \cdot P_{a b}^{c d}\left(\nabla_{c} \eta_{0}^{a} \nabla_{d} \eta_{0}^{b}-\nabla_{c} \eta_{*}^{a} \nabla_{d} \eta_{*}^{b}\right)\right.} \\
& \left.+T_{a b} \cdot\left[\eta_{0}^{a} \eta_{0}^{b}-\eta_{*}^{a} \eta_{*}^{b}\right]+\lambda(x) g_{a b} \cdot\left[\eta_{0}^{a} \eta_{0}^{b}-\eta_{*}^{a} \eta_{*}^{b}\right]\right]<0
\end{aligned}
$$

What this is saying is that there is no unique point, using this $\eta_{*}^{a}$ for which (4) holds. Therefore, we say there is no official point of instability of $\eta_{*}^{a}$ due to (3). The Lagrangian structure of what can be built up by the potentials given in (3) with respect to $\eta_{*}^{a}$ mean that we cannot expect an inflection point with respect to a $2^{\text {nd }}$ derivative of a potential system. Such an inflection point designating a speed up of acceleration due to DE exists a billion years ago [37]. Also note that the reason for the failure for (4) to be congruent to Fjoroft's theorem is due to

$$
\left[\frac{\partial^{2} U}{\partial\left(\eta^{a}\right)^{2}}=T_{a a}+\lambda(x) g_{a a}\right] \neq 0 \text {, for } \forall \eta_{*}^{a} \text { choices }
$$




\section{Appendix III. Details as to Forming Crowell's Time Dependent Wheeler De Witt} Equation, and Its Links to Worm Holes

This will be to show some things about the worm hole we assert the instanton traverses en route to our present universe. From Crowell [38]

$$
-\frac{1}{\eta r} \frac{\partial^{2} \Psi}{\partial r^{2}}+\frac{1}{\eta r^{2}} \cdot \frac{\partial \Psi}{\partial r}+r R^{(3)} \Psi=(r \eta \phi-r \ddot{\phi}) \cdot \Psi
$$

This has when we do it $\phi \approx \cos (\omega \cdot t)$, and frequently $R^{(3)} \approx$ constant, so then we can consider

$$
\phi \cong \int_{0}^{\infty} \mathrm{d} \omega\left[a(\omega) \cdot \mathrm{e}^{i k_{\omega} x^{\mu}}-a^{+}(\omega) \cdot \mathrm{e}^{-i k_{\sigma} x^{\mu}}\right]
$$

In order to do this, we can write out the following with regards to the solutions to Eqn (1) put up above.

$$
\begin{aligned}
C_{1}= & \eta^{2} \cdot\left(4 \cdot \sqrt{\pi} \cdot \frac{t}{2 \omega^{5}} \cdot J_{1}(\omega \cdot r)+\frac{4}{\omega^{5}} \cdot \sin (\omega \cdot r)+(\omega \cdot r) \cdot \cos (\omega \cdot r)\right) \\
& +\frac{15}{\omega^{5}} \cos (\omega \cdot r)-\frac{6}{\omega^{5}} \operatorname{Si}(\omega \cdot r)
\end{aligned}
$$

And

$$
C_{2}=\frac{3}{2 \cdot \omega^{4}} \cdot(1-\cos (\omega \cdot r))-4 \mathrm{e}^{-\omega \cdot r}+\frac{6}{\omega^{4}} \cdot \operatorname{Ci}(\omega \cdot r)
$$

This is where $\operatorname{Si}(\omega \cdot r)$ and $\operatorname{Ci}(\omega \cdot r)$ refer to integrals of the form $\int_{-\infty}^{x} \frac{\sin \left(x^{\prime}\right)}{x^{\prime}} \mathrm{d} x^{\prime}$ and $\int_{-\infty}^{x} \frac{\cos \left(x^{\prime}\right)}{x^{\prime}} \mathrm{d} x^{\prime}$. It so happens that this is for forming the wave functional permitting an instanton forming, while we next should consider if or not the instanton so farmed is stable under evolution of space time leading up to inflation. We argue here that we are forming an instanton whose thermal energy is focused into a wave functional which is in the throat of the worm hole up to a thermal discontinuity barrier at the onset, and beginning of the inflationary era.

\section{Appendix IV. The D’Albembertain Operation in an Equation of Motion for Emergent Scalar Fields}

We begin with the D'Albertain operator as part of an equation of motion for an emergent scalar field. We refer to the Penrose potential ( with an initial assumption of Euclidian flat space for computational simplicity) to account for, in a high temperature regime an emergent non zero value for the scalar field $\phi$ due to a zero effective mass, at high temperatures. [27]

When the mass approaches far lower values, it, a non zero scalar field re appears.

Leading to $\phi \underset{T \rightarrow 2.7 \text { Kelvin }}{\longrightarrow} \varepsilon^{+} \approx 0^{+}$as a vanishingly small contribution to cosmological evolution

Let us now begin to initiate how to model the Penrose quintessence scalar field evolution equation. To begin, look at the flat space version of the evolution equation

$$
\ddot{\phi}-\nabla^{2} \phi+\frac{\partial V}{\partial \phi}=0
$$

This is, in the Friedman-Walker metric using the following as a potential system to work with, namely:

$$
V(\phi) \sim-\left[\frac{1}{2} \cdot\left(M(T)+\frac{\mathfrak{R}}{6}\right) \phi^{2}+\frac{\tilde{a}}{4} \phi^{4}\right] \equiv-\left[\frac{1}{2} \cdot\left(M(T)+\frac{\kappa}{6 a^{2}(t)}\right) \phi^{2}+\frac{\tilde{a}}{4} \phi^{4}\right]
$$

This is pre supposing $\kappa \equiv \pm 1,0$, that one is picking a curvature signature which is compatible with an open universe.

That means $\kappa=-1,0$ as possibilities. So we will look at the $\kappa=-1,0$ values. We begin with. 


$$
\begin{aligned}
& \ddot{\phi}-\nabla^{2} \phi+\frac{\partial V}{\partial \phi}=0 \\
& \Rightarrow \phi^{2}=\frac{1}{\tilde{a}} \cdot\left\{c_{1}^{2}-\left[\alpha^{2}+\frac{\kappa}{6 a^{2}(t)}+M(T)\right]\right\} \\
& \Leftrightarrow \phi \equiv \mathrm{e}^{-\alpha \cdot r} \exp \left(c_{1} t\right)
\end{aligned}
$$

We find the following as far as basic phenomenology, namely

$$
\begin{aligned}
& \phi^{2}=\frac{1}{\tilde{a}} \cdot\left\{c_{1}^{2}-\left[\alpha^{2}+\frac{\kappa}{6 a^{2}(t)}+\left(M(T) \approx \varepsilon^{+}\right)\right]\right\} \underset{M(T \sim \text { high }) \rightarrow 0}{\longrightarrow} \phi^{2} \neq 0 \\
& \phi^{2}=\frac{1}{\tilde{a}} \cdot\left\{c_{1}^{2}-\left[\alpha^{2}+\frac{\kappa}{6 a^{2}(t)}+\left(M(T) \neq \varepsilon^{+}\right)\right]\right\} \underset{M(T \sim \text { Low }) \neq 0}{\longrightarrow} \phi^{2} \approx 0
\end{aligned}
$$

The difference is due to the behavior of $M(T)$. We use $M(T) \sim$ axion mass $m_{a}(T)$ in asymptotic limits with

$$
m_{a}(T) \cong 0.1 \cdot m_{a}(T=0) \cdot\left(\Lambda_{Q C D} / T\right)^{3.7}
$$

\section{Appendix V. Interesting Speculation. Does There Exist a Five Dimensional Version of an Instanton in the Worm Hole Transition Regime?}

We will attempt to build the contribution as to a Reissner-Nordstrom metric embedded in a five dimensional space-time metric, and see if this satisfied. i.e. look at (1) below This allows us to determine, using of the Risessner-Nordstrom metric as given, by Kip Thorne, Wheeler, and Misner [39], for an added cosmological "constant" $\Lambda$ and "charge" $Q$. This will be shown to lead to [40]

$$
\begin{aligned}
M_{g}(r) & =\int\left[T_{0}^{0}-\left(T_{1}^{2}+2 \cdot T_{2}^{2}\right)\right] \cdot \sqrt{-g_{4}} \mathrm{~d} V_{3} \\
& \approx \pi \cdot c_{1}^{2} \cdot\left[\frac{r^{3}}{3}-2 M \cdot \frac{r^{2}}{2}+Q \cdot r-\frac{\Lambda}{15} \cdot r^{5}\right]+4 \pi \cdot c_{1} \cdot\left[r^{2}-8 \cdot M \cdot r-\frac{\Lambda}{3} \cdot r^{4}\right] \\
& \underset{r \rightarrow \delta}{\longrightarrow} \varepsilon^{+} \approx 0
\end{aligned}
$$

To do this, we start off with the following space time line metric in five dimensions. This is a modification of Wesson's book. [40]

$$
\mathrm{d} S_{5-\operatorname{dim}}=[\exp (i \pi / 2)] \cdot\left\{\mathrm{e}^{2 \Phi(r)} \mathrm{d} t^{2}+\mathrm{e}^{2 \tilde{\Lambda}(r)} \mathrm{d} r^{2}+R^{2} \mathrm{~d} \Omega^{2}\right\}+(-1) \cdot \mathrm{e}^{\mu} \mathrm{d} l^{2}
$$

We claim that what is in the \{\} brackets is just the Reissner-Nordstrom line metric in four dimensional space. The parameters in the \{\} bracket are linked to the Reissner-Nordstrom metric via

$$
\mathrm{e}^{2 \Phi(r)}=\left(1-\frac{2 M}{r}+\frac{Q^{2}}{r^{2}}\right)
$$

And

$$
\mathrm{e}^{2 \tilde{\Lambda}(r)}=\left(1-\frac{2 M}{r}+\frac{Q^{2}}{r^{2}}\right)^{-1}
$$

And this is assuming that $R \sim r$ as well as using $\mu \approx c_{1} \cdot r$ with a maximum value topped off by a Planck's length value due to $\mu_{\text {Maximum }} \approx c_{1} \cdot r_{\text {Maximum }} \sim l_{P} \equiv 10^{-35} \mathrm{~cm}$. So being the case, we get the following stress tensor values 


$$
\begin{gathered}
T_{0}^{0}=\left(\frac{-1}{8 \pi}\right) \cdot\left(1-\frac{2 M}{r}+\frac{Q^{2}}{r^{2}}-\frac{\Lambda}{3} r^{2}\right) \cdot\left(\frac{c_{1}^{2}}{4}+\frac{c_{1}}{r}+\frac{c_{1}}{4} \cdot\left[\frac{\frac{2 M}{r^{2}}-\frac{2 Q}{r^{3}}-\frac{2 \Lambda r^{2}}{3}}{1-\frac{2 M}{r}+\frac{Q^{2}}{r^{2}}-\frac{\Lambda}{3} r^{2}}\right]\right) \\
T_{1}^{1}=\left(\frac{-1}{8 \pi}\right) \cdot\left(1-\frac{2 M}{r}+\frac{Q^{2}}{r^{2}}-\frac{\Lambda}{3} r^{2}\right) \cdot\left(\frac{c_{1}}{r}+\frac{c_{1}}{4} \cdot\left[\frac{\frac{2 M}{r^{2}}-\frac{2 Q}{r^{3}}-\frac{2 \Lambda r^{2}}{3}}{1-\frac{2 M}{r}+\frac{Q^{2}}{r^{2}}-\frac{\Lambda}{3} r^{2}}\right]\right) \\
T_{2}^{2}=T_{3}^{3}=\left(\frac{-1}{8 \pi}\right) \cdot\left(1-\frac{2 M}{r}+\frac{Q^{2}}{r^{2}}-\frac{\Lambda}{3} r^{2}\right) \cdot\left(\frac{c_{1}^{2}}{4}+\frac{c_{1}}{r}+\frac{c_{1}}{2} \cdot\left[\frac{\frac{2 M}{r^{2}}-\frac{2 Q}{r^{3}}-\frac{2 \Lambda r^{2}}{3}}{1-\frac{2 M}{r}+\frac{Q^{2}}{r^{2}}-\frac{\Lambda}{3} r^{2}}\right]\right)
\end{gathered}
$$

Furthermore, we get the following determinant value

$$
\sqrt{-g_{4}}=\left(1-\frac{2 M}{r}+\frac{Q^{2}}{r^{2}}-\frac{\Lambda}{3} r^{2}\right)
$$

All these together lead to (1) being satisfied. Let us now see how this same geometry contributes to a worm hole bridge and a solution as to forming the instanton flux wave functional between a prior to a present universe. The Reissner-Nordstrom metric permits us to have a radiation dominated 'matter' solution whose matter 'contribution' drops off rapidly as the spatial component of geometry goes to zero. This is in tandem with radiation pressure and density falling off rapidly, as we leave the center of such a purported soliton/ instanton. This is extremely useful because it ties in with the notion of fractional branes contributing to entropy calculations. In fact it is useful to state that these two notions dove tail with each other quite closely. The only difference is that the construction above does not in itself lend to the complexity of what we would observe, which is in itself a multiple-joined net work of charge centers and of shifting geometry.

\section{Appendix VI. Basic Physics of Achieving Minimum Precision in CMBR Power Spectra Measurements}

Begin first of all looking at

$$
\frac{\Delta T}{T} \equiv \sum_{l, m} a_{l m} Y_{l, m}(\theta, \phi)
$$

This leads to consider what to do with

$$
C_{l}=\left\langle\left|a_{l, m}\right|^{2}\right\rangle
$$

Samtleben et al. [31] consider then what the experimental variance in this power spectrum, to the tune of an achievable precision given by

$$
\frac{\Delta C_{l}}{C_{l}}=\sqrt{\frac{2}{2 l+1}} \cdot\left(\frac{1}{\sqrt{f_{\text {sky }}}}+\frac{4 \pi \cdot\left(\Delta T_{\text {exp }}\right)^{2}}{C_{l}} \cdot \sqrt{f_{\text {sky }}} \cdot \mathrm{e}^{l^{2} \sigma_{b}^{2}}\right)
$$

$f_{\text {sky }}$ is the fraction of the sky covered in the measurement, and $\Delta T_{\text {exp }}$ is a measurement of the total experimental sensitivity of the apparatus used. Also $\sigma_{b}$ is the width of a beam, while we have a minimum value of $l_{\min } \approx(1 / \Delta \Theta)$ which is one over the fluctuation of the angular extent of the experimental survey.

i.e. contributions to $C_{l}$ uncertainty from sample variance is equal to contributions to $C_{l}$ uncertainty from noise. The end result is

$$
4 \pi \cdot f_{\text {sky }}=C_{l} \cdot\left(\exp \left[-l^{2} \sigma^{2}\right]\right) /(\Delta T)^{2}
$$




\section{Appendix VII. Vacuum Fluctuations Which May Occur: Cosmological Perturbation Theory and Tensor Fluctuations (Gravity Waves)}

Durrer reviews how to interpret $C_{l}$ in the region where we have $2<l<100$, roughly in the region of the Sachs-Wolf contributions due to gravity waves. We begin first of all by looking at an initial perturbation, using a scalar field treatment of the 'Bardeen potential' $\Psi$ This can lead us to put up, if $H_{i}$ is the initial value of the Hubble expansion parameter

$$
k^{3}|\Psi|^{2} \cong\left(\frac{H_{i}}{M_{P}}\right)^{2}
$$

And

$$
\left\langle|\Psi|^{2}\right\rangle \cdot k^{3}=A^{2} k^{n-1} \cdot \eta_{0}^{n-1}
$$

Here we are interpreting $A=$ amplitude of metric perturbations at horizon scale, and we set $k=1 / \eta_{0}$, where $\eta$ is the conformal time, according to $\mathrm{d} t \equiv a \mathrm{~d} \eta=$ physical time, where we have $a$ as the scale factor.

Then for $2<l<100$, and $-3<n<3$, and a pure power law given by

$$
\left\langle|H(k, \eta=1 / k)|^{2}\right\rangle \cdot k^{3}=A_{T}^{2} k^{n_{T}} \cdot \eta_{0}^{-n_{T}}
$$

We get for tensor fluctuation, i.e. gravity waves, and a scale invariant spectrum with $n_{T}=0$

$$
C_{l}^{(T)} \approx \frac{A_{T}^{2}}{(l+3) \cdot(l-2)} \cdot \frac{1}{15 \pi}
$$

\section{Appendix VIII}

This is a direct quote from reference [28], and is put in which has the references [29]-[35] covered as reproduced from [28]. The remainder of this document concerns the matter of the LIGO and Virgo contributions to GW astronomy as seen in

\section{Beginning of quote from [28]}

4. Re-examining relic gravitational wave models as to what relic Gravitational waves could tell us about the origins of the early universe. As given in an earlier paper by the Author

Quoting from [9] we write the following. We reproduce this, because of the centrality of Equation (27) which is basic. It is very noticeable that in [10] we have that the following quote is particularly relevant to consider, in lieu of our results

\section{Quote}

"Thus, if advanced projects on the detection of GWs will improve their sensitivity allowing to perform a GWs astronomy (this is due because signals from GWs are quite weak) [1], one will only have to look the interferometer response functions to understand if General Relativity is the definitive theory of gravity. In fact, if only the two response functions (2) and (19) will be present, we will conclude that General Relativity is definitive. If the response function (22) will be present too, we will conclude that massless Scalar-Tensor Gravity is the correct theory of gravitation. Finally, if a longitudinal response function will be present, i.e. Equation (25) for a wave propagating parallel to one interferometer arm, or its generalization to angular dependences, we will learn that the correct theory of gravity will be massive Scalar-Tensor Gravity which is equivalent to $f(R)$ theories. In any case, such response functions will represent the definitive test for General Relativity. This is because General Relativity is the only gravity theory which admits only the two response functions (2) and (19) [4] [8]. Such response functions correspond to the two "canonical” polarizations $h+$ and $h \times$. Thus, if a third polarization will be present, a third response function will be detected by GWs interferometers and this fact will rule out General Relativity like the definitive theory of gravity”.

\section{End of quote}

We argue that a third polarization in Gravitational waves from the early universe may be detected, if there is proof positive that in the pre Planckian regime that the Corda conjecture [11] as given below, namely if the following analysis is part of our take on relic gravitational waves, is supported by the kinetic energy being larger than the potential energy, namely what if. 


\section{Quote}

"The case of massless Scalar-Tensor Gravity has been discussed in [4] [13] with a "bouncing photons analysis" similar to the previous one. In this case, the line-element in the TT gauge can be extended with one more polarization, labelled with $\Phi(\mathrm{t}+\mathrm{z})$, i.e. ...”.

End of quote: This ends our recap of the section given in [9] which we think is important

What we are arguing for is that the choice of the vacuum energy as given by Equation (27) may give conclusive proof as to satisfy the Corda conjecture and his supposition as to the existence of an additional polarization [10]. We will, in the future try to extend our results so as to determine if Equation (27) either falsifies or supports the existence of a $3^{\text {rd }}$ polarization. Which will be a way to determine the final disposition of GR as THE theory of Cosmology, or open up the possibility of alternate theories. It is an issue which we think will require extreme diligence. While ending our query as to the possible existence of a third polarization we should mention what would be the supreme benefit of our upcoming analysis of Equation (27), namely how to avoid the conflating of dust, with gravitational waves, i.e. the tragic Bicep 2 mistake [11]-[14].

End of quote from reference [29] which answers questions as to cosmological questions as to what is necessary for physics interpretation of both bicep 2, and the relative strength of polarization

Which indicate if we have a scalar-tensor theory of gravity, or something else as is discussed in [30]. We also need to avoid the problems alluded to in references [31] [32] which are due to Gravitational wave signals generated due to galactic dust, and can be seen to be due to multiple point sources in generated Gravitational wave signatures.

\section{End of quote from [28] from our present reference listing}

We now go to [31] directly. It is important that the information transfer and the mathematics thereof be in fidelity to requirements as of [31] directly. If they do not lead to falsifiable results pertinent to [32] then the supposition advanced in this paper are null and void and should be not continued. 